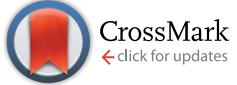

Cite this: RSC Adv., 2017, 7, 1326

Received 22nd November 2016 Accepted 6th December 2016

DOI: 10.1039/c6ra27141c

www.rsc.org/advances

\section{Preparation of freestanding graphene-based laminar membrane for clean-water intake via forward osmosis process $\dagger$}

\author{
Feng Yan, $\star^{\text {ab }}$ Chuhong Yu, $\star^{a}$ Bowu Zhang, ${ }^{\text {a }}$ Tao Zou, ${ }^{a}$ Hongwei Zhao ${ }^{a}$ \\ and Jingye $\mathrm{Li}^{* a}$
}

Here, a facile strategy is reported to prepare thin freestanding graphene oxide (GO) laminar membranes through thermally-induced chemical cross-linking process. The resultant freestanding cross-linked GO (F-CGO) membranes show controllable inter-laminar distance and hydraulic stability in aqueous media. Semi-permeability tests through a forward osmosis (FO) system show that the water permeation rate of the F-CGO membranes is up to $35.5 \mathrm{~L} \mathrm{~m}^{-2} \mathrm{~h}^{-1}$ when the draw solution is $2.0 \mathrm{~mol} \mathrm{~L}^{-1} \mathrm{NaCl}$ solution. This value is much higher than that of standing cross-linked GO (S-CGO) membranes, which indicates that the internal concentration polarization (ICP) effect has been alleviated by the F-CGO membrane. Furthermore, clean-water was taken in from various wastewaters, but without any detectable residual pollutants by using the F-CGO membrane as a separator. This work demonstrates that the F-CGO membrane is promising for water purification applications through the FO process, especially for portable water purification systems utilized during emergency situations.

\section{Introduction}

Recently, graphene oxide (GO) has emerged as an excellent membrane material, drawing huge attention from separation fields. Nair et al. demonstrated that sub-micrometre-thick laminar GO membranes allow for the un-impeded permeation of water, whereas they block everything else in the liquid or vapour form, and even gases including helium. ${ }^{1}$ They attribute these anomalous observations to the low-friction monolayer flow of water through the two-dimensional capillaries formed by the non-oxidized regions of the GO sheets. These capillaries block other molecules because they are narrowed or reversibly clogged with water. This work was then immediately expanded to explore the penetration behaviour of ions through GO membranes. Joshi et al. ${ }^{2}$ found that laminar GO membranes act as molecular sieves in aqueous solutions, blocking all solutes with a hydrated radii larger than $0.45 \mathrm{~nm}$, while the permeation rates of smaller ions through these membranes are thousands of times faster than those in simple diffusions. For the same reason, the network of

\footnotetext{
${ }^{a}$ Shanghai Institute of Applied Physics, Chinese Academy of Sciences, No. 2019 Jialuo Rd., Jiading Dist., Shanghai, 201800, China. E-mail: zhangbowu@foxmail.com; jingyeli@sinap.ac.an; Tel: +86-21-39194652

${ }^{b}$ University of Chinese Academy of Sciences, No. 19A, Yuquan Rd., Beijing, 100049, China

$\dagger$ Electronic supplementary information (ESI) available. See DOI: 10.1039/c6ra27141c

$\ddagger$ These authors contributed equally to this work.
}

nanocapillaries only accepts species that fit in, and a capillarylike high pressure acts on ions inside the capillaries, accelerating their diffusion behaviour. ${ }^{3}$ The manifested sizeexclusion properties make GO laminar membranes promising materials for ion-selective separation, ${ }^{4-6}$ organic-water separation,,$^{7-9}$ water desalination ${ }^{10-12}$ and purification, ${ }^{13-15}$ and so on. However, owing to the highly hydrophilic and negatively charged oxygen-containing groups of the GO sheets, ${ }^{16}$ the inter-layer distance ( $d$-spacing) of laminar GO membranes will be enlarged, causing the possible disintegration of the two-dimensional capillaries when they are immersed in aqueous media for long time periods. ${ }^{17}$ That is why a pure GO laminar membrane cannot effectively perform its size-exclusion function during membrane separation processes due to its low mechanical stability. ${ }^{16,18,19}$ Therefore, to enhance their stability in aqueous systems, laminar GO membranes need to be modified by processes such as chemical cross-linking, ${ }^{20-22}$ partial reduction ${ }^{23,24}$ and intercalation. ${ }^{25}$

As an emerging membrane technology, forward osmosis (FO) has attracted much attention because of its wonderful advantages over other traditional membrane separation techniques, including low energy consumption, ${ }^{26}$ low membrane fouling $^{27-29}$ and low equipment requirement, and so on. Nevertheless, the application of FO technique still suffers from some problems, especially, internal concentration polarization (ICP) ${ }^{26}$ which stems from the bilayer structure of $\mathrm{FO}$ membrane, thin selective layer and porous supporting layer. ${ }^{30}$ The severe ICP effect during FO process would induce the 
driving force decrease, further reduce the membrane flux. In principle, FO process is based on the natural osmosis phenomenon, in which water is drawn across a semipermeable membrane from a feed solution into a draw solution by an osmotic pressure difference $(\Delta \pi)$ between the two solutions, while the solutes are left behind in the feed solution. The FO membrane is just required to stand the osmotic pressure, which is much lower than membranes for reverse osmosis (RO), nanofiltration (NF), or ultrafiltration (UF) process. Therefore, freestanding FO membrane, without the supporting layer, has been proposed to eliminate ICP effect in recent. ${ }^{23}$

Taking into consideration the excellent size-exclusion, water permeation, and outstanding mechanical strength properties of laminar GO membranes, ${ }^{31,32}$ they are promising materials for the fabrication of freestanding semipermeable membranes for FO processes. For instance, ultrathin laminar GO membranes (40-200 $\mathrm{nm})$, reduced by hydrogen iodide (HI) vapor, exhibited a high enough tensile strength (170-270 MPa) for FO processes. In addition, the obtained freestanding reduced GO membranes showed a higher salt rejection and water permeation than commercial cellulose triacetate (CTA) FO membranes. ${ }^{23}$ Sun et al. prepared a freestanding hybrid membrane consisting of anionic $\mathrm{GO}$, cationic $\mathrm{Co}-\mathrm{Al}$ (or $\mathrm{Mg}-\mathrm{Al}$ ) layered double hydroxide (LDH) that exhibited robust mechanical strength, and a highly selective charge-guided ion transport in concentration gradientdriven systems. ${ }^{25}$

In this work, we report a facile strategy to prepare thin freestanding graphene-based laminar membrane through thermally-induced chemical cross-linking process, named as freestanding crosslinked GO (F-CGO) membranes. The sensitivity of water to the inter-laminar distance of the F-CGO membranes was evaluated by X-ray diffraction (XRD), and terahertz time-domain spectroscopy (THz-TDS). The salt rejection performance and ICP effects of these F-CGO membranes were investigated in a custom-made FO system, using $\mathrm{NaCl}$ solution as the draw solution and ultrapure water as the feed solution. In addition, the F-CGO membrane was used to intake clean-water from various wastewaters that contained heavy metal ions or organic dyes.

\section{Experimental}

\section{Preparation of F-CGO membranes}

The monolayer GO sheets were prepared using modified Hummer's method as reported in our previous works. ${ }^{33-35}$ Details are shown in the ESI. $\dagger$ The scheme of the F-CGO membranes preparation was shown as Scheme 1, and the details could be described as follows. $10 \mathrm{~mL}$ of GO $\left(0.5 \mathrm{mg} \mathrm{mL} \mathrm{mL}^{-1}\right) / \mathrm{PA}$ (propanedioic acid) mixtures, with different weight ratios of GO/PA, were prepared and mildly shaken for a few minutes. Then, these solutions were poured into the glass cup of a vacuum filter with a CA microfiltration membrane (pore size, $0.22 \mu \mathrm{m}$ ). When a filtration cake was formed on the CA membrane by continuous pumping, several droplets of $\mathrm{HCl}$ solution $\left(\sim 9.6 \mathrm{~mol} \mathrm{~L}^{-1}\right)$ were distributed on the cake, and then pump-filtrated until dry. After that, the CA membrane coated with GO sheets was taken off from the filter, and infiltrated with another several droplets of the $\mathrm{HCl}$ solution. Then, to make sure the cross-linking reaction was carried out, the GO sheets coated CA membrane was put in an oven at $120{ }^{\circ} \mathrm{C}$ for $30 \mathrm{~min}$. The cross-linking reaction involves the esterification of the carboxylic acid on the PA with the hydroxyl groups of the GO sheets. After cross-linking, this standing crosslinked GO layer on CA membrane was named S-CGO membrane, while the F-CGO membrane was obtained by mechanically peeling off the cross-linked GO layer from the CA microfiltration membrane. As a contrast, a GO membrane without PA addition was also prepared by the same procedure and labelled as GO-120 membrane. The areal loading of GO sheets was about $0.35 \mathrm{mg} \mathrm{cm}^{-2}$ in all of here prepared thin freestanding GO-based membranes.

\section{Semi-permeation tests}

The semi-permeation tests were carried out on a custom-made FO permeation cell as show in Fig. S1. $\dagger$ The permeation cell consisted of two glass compartments, separated by the F-CGO membrane, with a $19.6 \mathrm{~mm}^{-2}$ contact area. One compartment was filled with ultrapure water as the feed solution, and the other compartment was filled with an aqueous $\mathrm{NaCl}$ solution, at
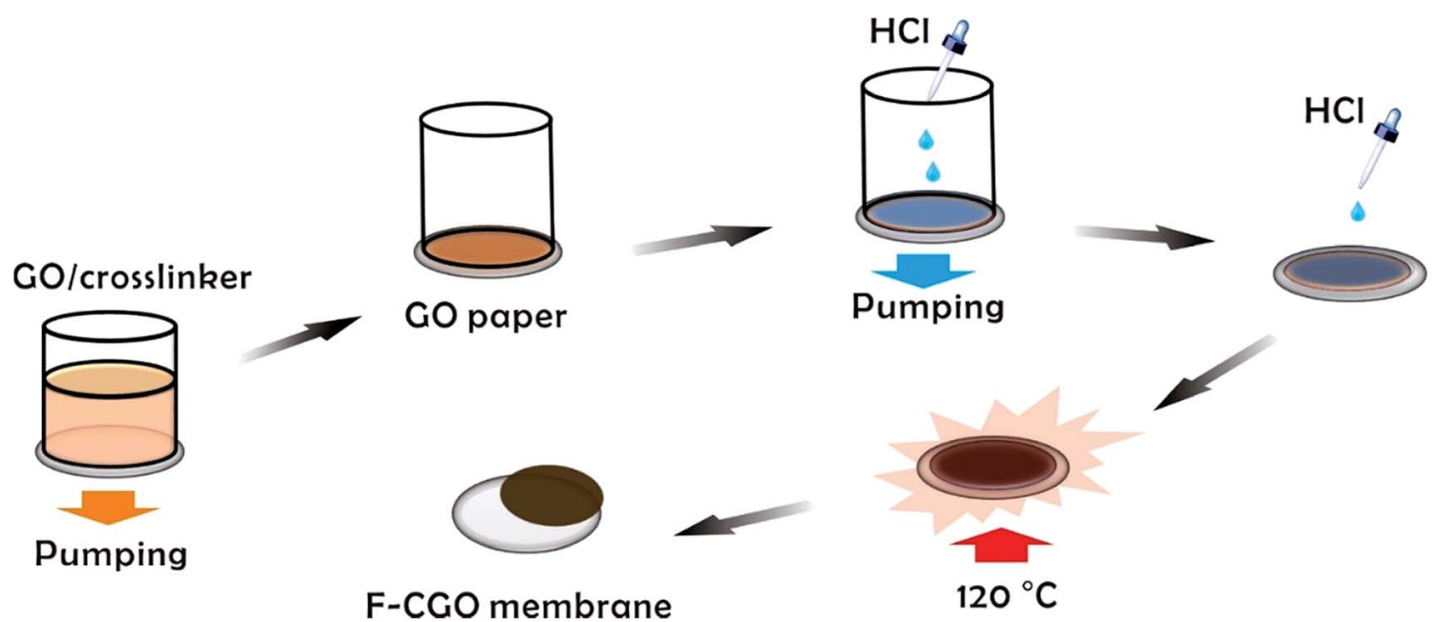

Scheme 1 Schematic diagram of the F-CGO membranes preparation process. 
a pre-set concentration, as the draw solution. The feed solution was circulated between the corresponding compartment of FO permeation cell and an Erlenmeyer flask using a peristaltic pump with a $45 \mathrm{~mL} \mathrm{~min}^{-1}$ flow rate, and so was the draw solution. The initial volume of the feed solution was equal to that of the draw solution. The Erlenmeyer flask-stored draw solution was weighed on an electronic balance during the permeation tests, and this weigh was used to calculate the water permeation rate of the membrane using the following equation:

$$
R_{\mathrm{w}}=\frac{W_{t+1}-W_{t}}{S_{\mathrm{m}} \Delta t},
$$

where $R_{\mathrm{W}}$ is the water permeation rate; $W_{t}$ and $W_{t+1}$ are the weight of the draw solution at sampling time points $(t)$ and $(t+$ 1), respectively; $\Delta t$ is the time interval between two sampling points; and $S_{\mathrm{m}}$ is the effective contact area of the membrane between the two compartments of the permeation cell. At the same time, a few millilitres of the feed solution were sampled and determined by Inductively Coupled Plasma Atomic Emission Spectrometry (ICP-AES) to measure the reverse salt rate, which was calculated as follows:

$$
R_{\mathrm{s}}=\frac{C_{t+1} V_{t+1}-C_{t} V_{t}}{S_{\mathrm{m}} \Delta t}
$$

where $R_{\mathrm{S}}$ is the reverse salt rate; $C_{t}$ and $C_{t+1}$ are the concentrations of $\mathrm{Na}^{+}$at sampling time points $(t)$ and $(t+1)$, respectively; $V_{t}$ and $V_{t+1}$ are the volume of the feed solution at sampling time points $(t)$ and $(t+1)$, respectively. To have an indication of the membrane's $\mathrm{Na}^{+}$rejection performance, we calculated the $\mathrm{Na}^{+}$ selective permeation coefficient across the membrane based on the water and $\mathrm{Na}^{+}$ion permeation rates, as follows:

$$
S=\frac{C_{\mathrm{d}} V_{\mathrm{d}}}{C_{\mathrm{f}} V_{\mathrm{f}}},
$$

where $S$ is the selective permeation coefficient of $\mathrm{Na}^{+} ; C_{\mathrm{d}}$ and $V_{\mathrm{d}}$ are the $\mathrm{Na}^{+}$concentration and volume of draw solution after $4 \mathrm{~h}$ permeation, respectively; and $C_{\mathrm{f}}$ and $V_{\mathrm{f}}$ are the $\mathrm{Na}^{+}$concentration and volume of feed solution after $4 \mathrm{~h}$ permeation period, respectively. Therefore, the rejection of $\mathrm{Na}^{+}$could be calculated as follows:

$$
R_{\mathrm{J}}=\frac{S}{S+1},
$$

where $R_{\mathrm{J}}$ is the $\mathrm{Na}^{+}$rejection of the F-CGO membrane after $4 \mathrm{~h}$ permeation.

\section{Clean-water intake from wastewaters}

The setup and procedure of this test are similar to those of the semi-permeation test, except for the feed and draw solutions. For this test, the feed solution was composed of a $10 \mathrm{ppm}$ methylene orange (MO), rhodamine $\mathrm{B}(\mathrm{RB})$, or $\mathrm{Cu}^{2+}$ aqueous solution; and the draw solution consisted of a $2.0 \mathrm{~mol} \mathrm{~L}^{-1} \mathrm{NaCl}$ solution. The water permeation rate in the different feed solutions was determined by measuring the weight change of the draw solution. The draw solution was monitored by UV-Vis spectroscopy (MO or RB feed solution), or with ICP-AES instruments, to detect the leaking pollutants during the test.

\section{Results and discussion}

\section{Preparation of F-CGO membranes}

The GO sheets prepared using modified Hummer's method, were monolayer and had a basal size of about $10 \mu \mathrm{m}$ (Fig. S2 $\dagger$ ). PA was used as the cross-linker because of its acid-assisted esterification with the hydroxyl groups on the GO sheet. ${ }^{21}$ As the Scheme 1 shows, after $\mathrm{HCl}$ solution wetting and a $120{ }^{\circ} \mathrm{C}$ heat treatment, the GO-PA paper was easily released from the supporting cellulose acetate (CA) membrane (Fig. 1a). The obtained F-CGO membranes with different cross-linking degrees were prepared by adjusting the PA/GO weight ratio and labelled as F-CGO $x$ membranes, where the $x$ denotes the PA/GO weight ratio in the corresponding GO/PA mixture dispersion used for membrane preparation. The thermogravimetric analysis (TGA) curves of the F-CGO membranes show the weight loss from $130{ }^{\circ} \mathrm{C}$ to $210{ }^{\circ} \mathrm{C}$ was increased as the PA/GO weight ratio increasing (Fig. S3 $\dagger$ ), which confirms the cross-linking degree of F-CGO membrane increased, because of the poorer thermal stability of cross-linker than that of GO sheets. We also controlled the thickness of the F-CGO membranes by adjusting the dosage of the GO dispersion. As it is shown in the insert of Fig. 1a, the resultant F-CGO3 membrane exhibits semitransparency, and the cross-sectional SEM image shows its thickness to be about $1 \mu \mathrm{m}$, with a noticeable regular laminar structure (Fig. 1b). Fig. 1c shows that the surface of the F-CGO membrane appears to have a ridge-like morphology, which can be explained by the unevenly stress applied on the GO sheets during their pumping-assistance assembly. This deduction is supported by the reverse surface morphology and optical microscopy images of the F-CGO membrane (Fig. S4 $\dagger$ ), in which the observed radial trends of the ridges reflect that the strong suction at the micro-pores of the supporting membrane resulted in the uneven assembly of the GO sheet.

The FT-IR spectra of different GO membranes show that the $\mathrm{C}=\mathrm{O}\left(\sim 1730 \mathrm{~cm}^{-1}\right)$ and $\mathrm{C}-\mathrm{O}\left(1300-1050 \mathrm{~cm}^{-1}\right)$ absorption bands increased after cross-linking (Fig. 1d). This behaviour is explained by the esterification of the GO hydroxyl groups with the carboxylic acid of the PA. ${ }^{21}$ Investigating the C1s XPS spectra of the F-CGO membrane and GO paper after their drying at room temperature $\left(25{ }^{\circ} \mathrm{C}\right.$ ) and $120{ }^{\circ} \mathrm{C}$ (labelled as GO-25 and GO-120, respectively), we found that the intensity of peak at $\sim 284.6 \mathrm{eV}(C-\mathrm{C} / C-\mathrm{H})$ increased obviously, and the peak at $\sim 288.8 \mathrm{eV}(C=\mathrm{O})$ decreased after $120{ }^{\circ} \mathrm{C}$ treatment whether PA addition or not (Fig. 2), which demonstrates the GO sheets were partially reduced during the thermal treatment. Comparing with the GO-120 membrane, as shown in the Fig. $2 \mathrm{c}$ and $\mathrm{d}$, the intensity of the peak at $\sim 286 \mathrm{eV}$, assigned as the $\mathrm{C}-\mathrm{OH}$ signal, ${ }^{36}$ decreased sharply; while the intensity of the $C-\mathrm{O}-\mathrm{C}=\mathrm{O} / \mathrm{C}-\mathrm{O}-C$ $(\sim 287.2 \mathrm{eV})^{36}$ peak increased. These results confirm that chemical cross-linking happened between the PA and GO sheets via esterification. Moreover, the higher area ratio of $C-\mathrm{O}-\mathrm{C}=\mathrm{O}$ / $\mathrm{C}-\mathrm{O}-\mathrm{C}$ signal in F-CGO3 membrane (Fig. 2d) indicates the (Fig. 2c) higher cross-linking degree than that of F-CGO1 membrane, which is evidenced by the pyrolysis behaviour of as-prepared F-CGO membranes from different PA/GO 

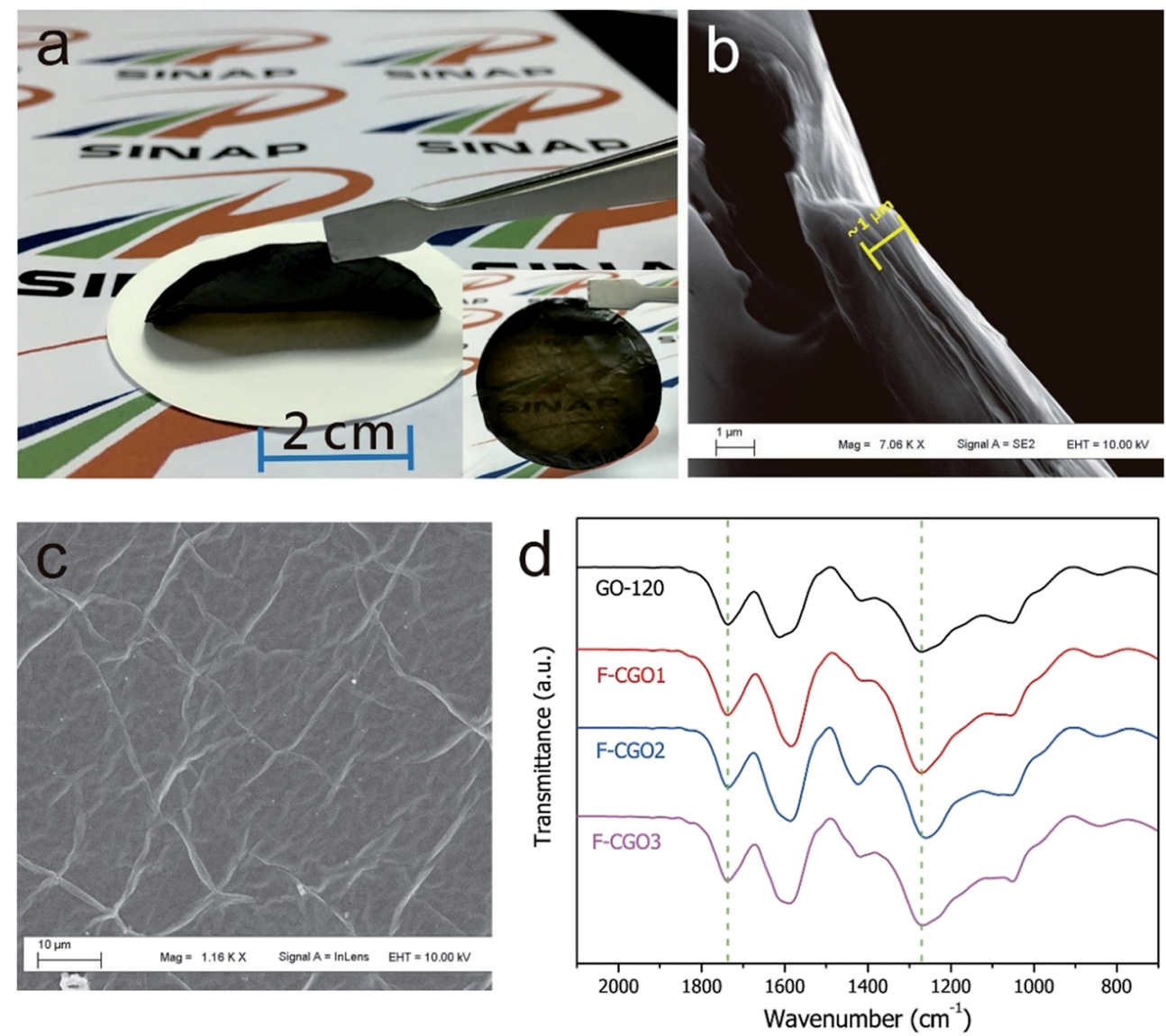

Fig. 1 (a) Digital pictures of the F-CGO3 membrane and its removal from the supporting membrane. (b) Cross-section and (c) surface micromorphology of the F-CGO3 membrane. (d) FT-IR spectra of the GO-120 membrane and different F-CGO membranes, which were prepared using different cross-linker dosages (the numbers denote the weight ratio of PA/GO in the mixture dispersion), which were tested on transmission mode. The scale bar in (b) and (c) was $1 \mu \mathrm{m}$ and $10 \mu \mathrm{m}$, respectively.

dispersions (Fig. S3†). The Raman spectra of the GO-120 and FCGO3 membranes show that the $I_{\mathrm{D}} / I_{\mathrm{G}}$ ratio increased slightly after cross-linking, which is in accordance with that was previously reported, indicating that only small distortions were introduced on the laminar structure after cross-linking (Fig. S5 $\dagger$ ). ${ }^{37}$ Interestingly, the $I_{2 \mathrm{D}} / I_{\mathrm{S} 3}$ ratio was slightly increased from 0.609 of GO-120 to 0.694 of F-CGO3, where the $\mathrm{S} 3$ peak is a second-order peak due to the $\mathrm{D}-\mathrm{G}$ combination, and $2 \mathrm{D}$ refers to the graphene $2 \mathrm{D}$ peak ${ }^{38}$ which indicates that less topological disorder within GO sheets in F-CGO3 membrane. ${ }^{39}$ In other words, the defect density of GO sheets in the F-CGO3 membranes was further reduced during the thermal treatment at $120{ }^{\circ} \mathrm{C}$ with the assistance of crosslinker,,$^{38}$ which was accompanied by a higher intensity of C-C/ $\mathrm{C}-\mathrm{H}$ peak compared to the GO-120 membrane (Fig. 2). Additionally, it would also indicate the cross-linking between adjacent GO sheets enhanced the structure order of GO-based membrane, which gives another reason for the light change of $D$ peaks in Raman spectra after cross-linker introduction.

\section{Water sensitivity and hydraulic stability}

In Fig. 3a, the water contact angle of the pristine GO membrane (air-drying at $25^{\circ} \mathrm{C}, \mathrm{GO}-25$ ) was $\sim 40^{\circ}$. This value increased to $>70^{\circ}$ after the membrane was oven-dried at $120{ }^{\circ} \mathrm{C}$ or crosslinked with PA, which indicates the partial reduction of GO sheets in the membranes after these treatments. This result is in accordance with the Raman spectra of the GO-120 and FCGO3 membranes. The partial reduction and increased hydrophobicity of the F-CGO3 membrane could enhance its structural stability in aqueous media. The XRD patterns of the dry membranes (Fig. 3b and S6a $\dagger$ ) show that the (002) diffraction peak increased from $10.2^{\circ}$ (GO-25, $d$-spacing $0.861 \mathrm{~nm}$ ) to $10.4^{\circ}$ (GO-120, $d$-spacing $0.852 \mathrm{~nm}$ ) after the heat treatment, indicating the achievement of a more compact laminar structure. It is noted that the diffraction peaks were further increased to $10.5^{\circ}$ ( $d$-spacing $\left.0.844 \mathrm{~nm}\right), 10.8^{\circ}(d$-spacing $0.814 \mathrm{~nm})$ and $10.9^{\circ}$ ( $d$-spacing $0.81 \mathrm{~nm}$ ), as the PA/GO weight ratio increased. This suggests that the cross-linking between PA and GO does not expand, but narrow the interlayer distance instead. This behaviour could be explained that the small size of cross-linker molecule and low cross-linking degree of PA (as shown by the TGA curves in Fig. S3 $†$ ), are unable to undermine the GO sheets stacking, which happens during the polymer decoration of GO sheets. ${ }^{36}$ Through comparisons of the XRD patterns from the wet state of the membranes (Fig. $3 \mathrm{~b}$ and S6b $\dagger$ ), it was observed that the GO-25 diffraction broadens and its $2 \theta$ angle shifts to 

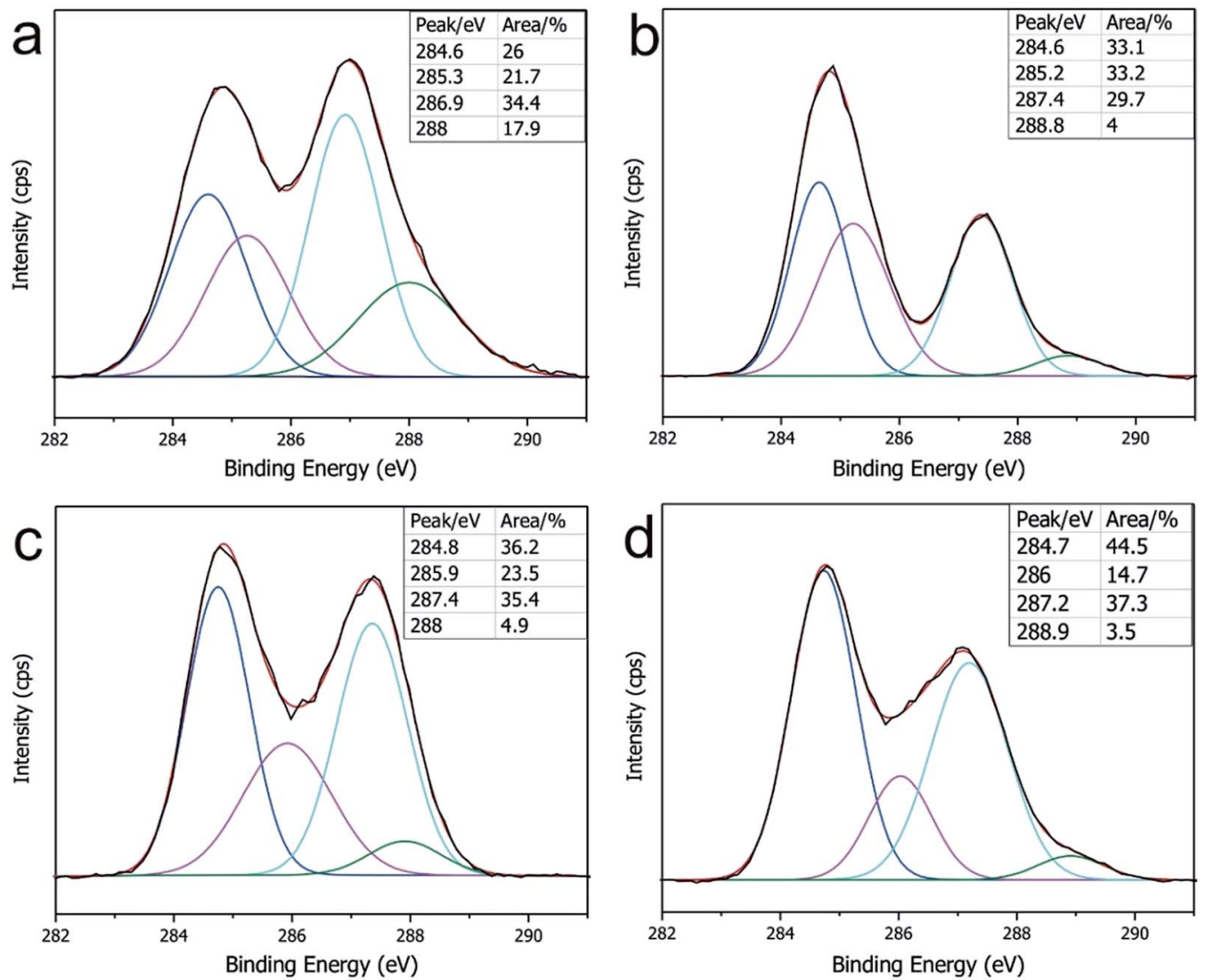

Fig. 2 The C1s XPS spectra of the (a) pristine GO membrane (GO-25), (b) GO-120 membrane, (c) F-CGO1 membrane and (d) F-CGO3 membrane.

$6.6^{\circ}$ ( $d$-spacing $\left.1.34 \mathrm{~nm}\right)$, while the other membranes still keep sharp peak curves, even though their $2 \theta$ angles shift to at least $7.0^{\circ}$ (d-spacing $1.25 \mathrm{~nm}$ ).

The effect of water on the structure of the membranes was monitored by terahertz time-domain spectroscopy (THz-TDS), which is a novel and promising tool to characterize the weak interactions of molecules, such as hydrogen bond and van der Waals force, because of its unique time-resolved feature and high signal to noise (SNR) in THz frequency range (wavelength in $0.03-3 \mathrm{~mm}) .^{40}$ As a polar liquid, water strongly absorbs $\mathrm{THz}$ radiation, which enables the THz-TDS to determine the absorption coefficient and refractive index of aqueous sample in THz range. ${ }^{41,42}$ The THz-TDS spectra of GO-25, GO-120 and FCGO3 membranes containing different water contents were recorded and shown in Fig. 3c, d and S7a. $\uparrow$ Due to the highly sensitive to water, the THz-absorbance of the GO-25, GO-120 and F-CGO3 membranes (Fig. S7b-d $\dagger$ ) from 0.5 to $3.5 \mathrm{THz}$ demonstrates a postive relationship with the water contents in the GO-25, GO-120 and F-CGO3 membranes. The THz-TDS spectra show that the transmission intensity of the dry FCGO3 membrane is slightly lower than that of the dry GO-25 and GO-120 membrane because of the introduction of crosslinker into the F-CGO3 membrane, which would be THzsensitive and thus bring about the transmittance decreasing of incent $\mathrm{THz}$ radiation. This result is very consistent with those from FT-IR, Raman and TG analyses. A noticeable time delay can be observed in the THz-TDS spectra when the membrane was at different wet states. The time delay was obvious higher for the F-CGO3 membrane than that for the GO-25 membrane (Fig. 3c and d). In principle, the time delay is the function of the refractive index and thickness of sample material, and can be defined mathematically as follows: ${ }^{43}$

$$
\Delta t \approx \frac{\left(n_{2}-n_{1}\right) d}{c}
$$

where $\Delta t$ is the time delay degree; $c$ is the speed of light; $d$ is the thickness of the sample; and $n_{2}$ and $n_{1}$ are the refractive indexes of the material and background, respectively. Dry air was taken as the background in our experiment.

As eqn (5) shows, the time delay degree is positively related to the refractive index and thickness of the membrane. After being wetted with water, the refractive index and thickness of the GO membranes simultaneously increase. Given the higher water stability of the F-CGO3 membrane, the thickness of the wet FCGO3 membrane should not be larger than that of the wet GO-25 membrane. Thereby, the refractive index of the wet FCGO3 membrane should be larger than that of the wet GO-25 membrane. In fact, we calculated the refractive index of GO25 and F-CGO3 membrane at dry and wet state by the TAS7400 system software and the arithmetic library provided from the $\mathrm{THz}$ instruments manufacture (Advantest 

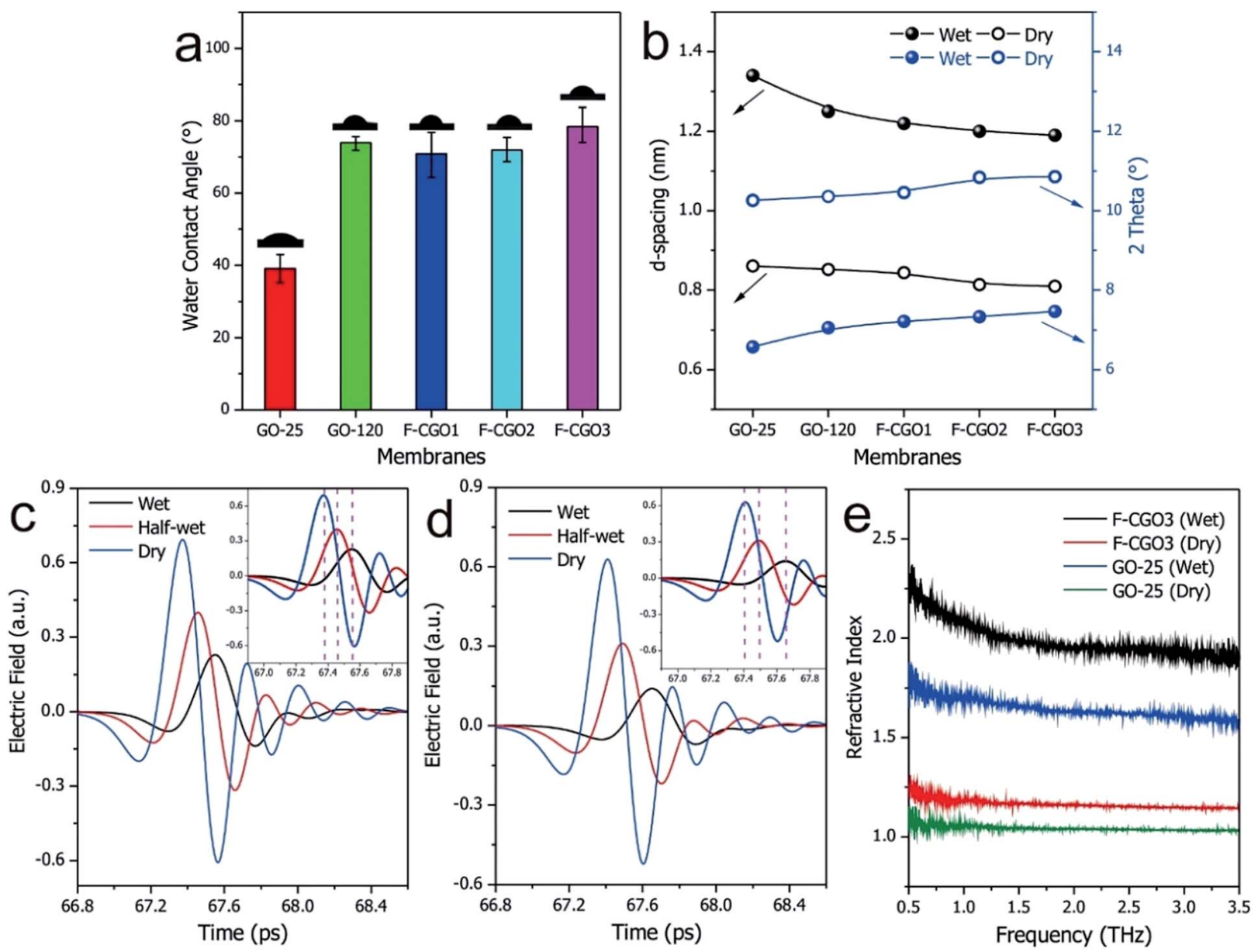

Fig. 3 (a) Water contact angle on different GO membranes surface; (b) comparison of the $d$-spacing of different GO membranes at the dry and wet states (immersed in water for $1 \mathrm{~h}$ ); and THz-TDS spectra of (c) GO-25 and (d) F-CGO3 membranes at different wet states. (e) The refractive indexes of the GO-25 and F-CGO3 membranes at wet and dry states. Dry state: stored in $25^{\circ} \mathrm{C}$ desiccator overnight; wet state: picked out from ultrapure water after immersing $1 \mathrm{~h}$ and blotted with paper; half-wet state: leaving in sample chamber of THz spectrometer for 10 min.

Corporation, Japan). As Fig. 3e shows, the refractive indexes of both GO-25 and F-CGO3 membranes are increased obviously after water wetting, but the refractive index of wet F-CGO3 membrane is really larger than that of wet GO-25 membrane. As we know, the refractive index is relevant for the material density. ${ }^{44,45}$ Therefore, our results indicate that the density of wet F-CGO3 membrane is higher than that of the wet GO-25, which is a result of the cross-linking treatment having enhanced the GO sheets stacking, and thus producing a more stable membrane in water.

The obtained F-CGO3 membrane was cut into $0.8 \times 3.0 \mathrm{~cm}$ strips, and hung on the bracket of a retort stand. The strips exhibited good strength as they could withstand at least a $20 \mathrm{~g}$ weight and $2 \mathrm{~g}$ aids (Fig. S8 ${ }^{\dagger}$ ). To assess the hydraulic stability of the membranes visually, the maximum hydraulic pressure that the membranes could resist in the FO setup was also measured. The F-CGO membranes with $1 \mu \mathrm{m}$ thickness (attached on a silicone pad with an aperture of $19.6 \mathrm{~mm}^{-2}$, as shown in Fig. S9a†) were able to withstand static hydraulic pressures up to $\sim 9 \mathrm{kPa}$, much higher than that of the GO-120 membrane (Fig. S10b†), which indicates the cross-linking treatment enhanced the hydraulic stability of GO membrane obviously. It also was shown that the hydraulic stability of F-CGO membranes is proportional to their crosslinking degree, which means that the GO sheets in the membrane were tightly connected together by cross-linker, and formed a consubstantial entity. This result demonstrates the F-CGO membranes would be able to withstanding osmotic pressure during FO process.

\section{Semi-permeability of F-CGO membranes}

Taking into consideration the high osmotic potential and nontoxicity of $\mathrm{NaCl}$, it is an ideal and promising candidate as a hydration draw reagent for the FO setup. Here, we mainly investigated the $\mathrm{Na}^{+}$permeability of F-CGO membranes by using a custom-made osmotic cell, as shown in Fig. S1. $\dagger$ In this set-up, one side of the osmotic cell was ultrapure water (the feed solution), and the other side was the $\mathrm{NaCl}$ solution (the draw solution). Fig. 4 a shows that when the concentration of the $\mathrm{NaCl}$ solution was $0.5 \mathrm{~mol} \mathrm{~L}^{-1}$, the $\mathrm{Na}^{+}$permeation decreased as the cross-linking degree increased, which is in accordance with the $d$-spacing changes observed after cross-linking (Fig. 3b). We 

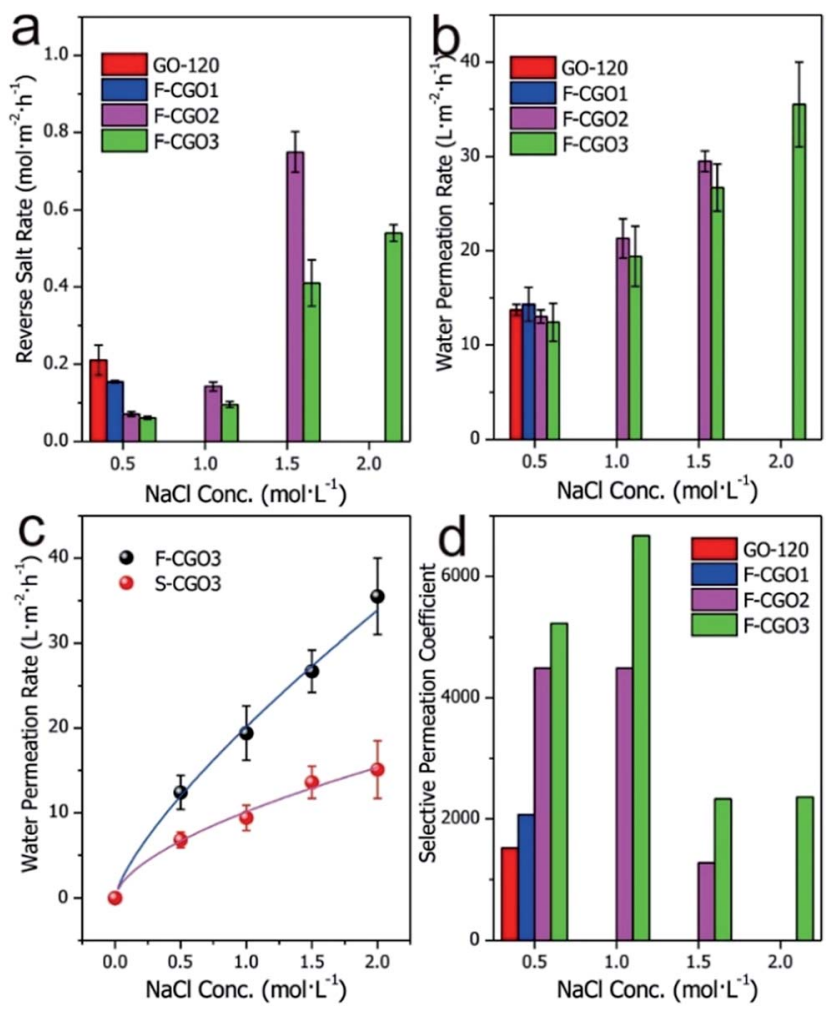

Fig. 4 (a) Reverse salt diffusion and (b) water permeation results of different GO membranes; (c) comparison between the water permeation of F-CGO3 and S-CGO3 membranes; (d) selective $\mathrm{Na}^{+}$permeation coefficient across different GO membranes. The feed solution was ultrapure water, and the draw solution was a $2.0 \mathrm{~mol} \mathrm{~L}^{-1} \mathrm{NaCl}$ solution. The active layer of the S-CGO membrane faced the feed solution. The reverse salt diffusion test lasted $4 \mathrm{~h}$, while the water permeation rate was obtained from a $0.5 \mathrm{~h}$ permeation period.

also found that F-CGO membranes with different crosslinking degrees exhibited different swelling durability in high salinity solutions. When a $1.0 \mathrm{~mol} \mathrm{~L}^{-1} \mathrm{NaCl}$ solution was used as the draw solution, the GO-120 and F-CGO1 membranes disintegrated after several minutes of testing. As the $\mathrm{NaCl}$ concentration increased to $2.0 \mathrm{~mol} \mathrm{~L}^{-1}$, the $\mathrm{F}-\mathrm{CGO} 2$ membrane also experienced disintegration. This behaviour is explained by the high osmotic pressure induced on the GO membranes by the highly concentrated draw solution. These results indicate that the higher crosslinking degree of F-CGO membranes not only hinders the diffusion of $\mathrm{Na}^{+}$, but it also provides the membrane with a good structural stability in highly saline water, which is in accordance with the static hydraulic pressure test results (Fig. S9b†).

Based on the comparison of $\mathrm{Na}^{+}$permeation through crosslinked GO membranes with and without a supporting CA microfiltration membrane (Fig. S10†), it was found that the $\mathrm{Na}^{+}$ permeation of the F-CGO3 membrane was higher than that of the S-CGO3 membrane. Given the same structure and thickness of the active layer of the S-CGO3 membrane with that of the FCGO3 membrane, the effect of the porous supporting membrane is accountable for the differences in the $\mathrm{Na}^{+}$ permeation. As previously mentioned, the porous supporting membrane decreases the osmotic gradient decrease because it concentrates the solutes in the supporting layer (i.e. ICP effect). Thus, a decreasing driving force results in a decreasing $\mathrm{Na}^{+}$ permeation rate through the crosslinked GO membrane, which indicates that the permeation behaviour of the S-CGO membrane suffers from serious ICP effects, in comparison to the F-CGO membrane, because of its porous supporting layer.

The water permeation of different membranes is presented in Fig. $4 \mathrm{~b}$, where it can be observed that the water permeation of FCGO membranes is inversely proportional to their cross-linking degree, although it does not differ much for the different samples. Even when a highly concentrated draw solution was used, there was only a slight difference between the water permeation values of the F-CGO2 and F-CGO3 membranes, which may be due to differences in the $d$-spacing of GO membranes after they were cross-linked under different PA dosages. This indicates that cross-linking the GO sheets has little impact on the water permeation of the membrane. Fig. 4c shows that the water permeation of the F-CGO3 membrane is higher than that of the SCGO3 membrane, particularly when using a highly concentrated draw solution. This result confirms that the freestanding membrane (F-CGO) is better for water permeation than the composite membrane (S-CGO) because, even though it has a faster cross-membrane diffusion of the draw reagent (Fig. S10†), the ICP effects on the freestanding membrane were efficiently alleviated or eliminated. We also noted that the water permeation rate of the F-CGO3 membrane was $\sim 35 \mathrm{~L} \mathrm{~m}^{-2} \mathrm{~h}^{-1}$ when using a $2.0 \mathrm{~mol} \mathrm{~L}^{-1} \mathrm{NaCl}$ solution as the draw solution, and ultrapure water as the feed solution. This value is about three times that of commercial cellulose triacetate (CTA) FO membranes produced by HTI Inc. under the same testing conditions. ${ }^{23}$

To further reveal the semi-permeability of the F-CGO membrane, we calculated the selective $\mathrm{Na}^{+}$permeation coefficient across the membranes based on the reverse salt rate and water permeation rate. This parameter is defined as the molar ratio of $\mathrm{Na}^{+}$in the draw solution to that in the feed solution after permeation testing; and it indicates the $\mathrm{Na}^{+}$rejection performance of the membranes under different conditions. Fig. 4d shows that when the $\mathrm{NaCl}$ concentration of the draw solution is $0.5 \mathrm{~mol} \mathrm{~L}^{-1}$, the $\mathrm{Na}^{+}$rejection performance increases as the cross-linking degree increases, which is a trend in agreement with the reverse salt diffusion behaviour of different membranes (Fig. 4a). The F-CGO3 membrane showed the highest $\mathrm{Na}^{+}$rejection value of $\sim 6700\left(1.0 \mathrm{~mol} \mathrm{~L}^{-1} \mathrm{NaCl}\right.$ solution as the draw solution). Even at the highest saline solution, the $\mathrm{Na}^{+}$selective permeation coefficient was higher than 2000, which means that the F-CGO3 membrane rejected $99.95 \%$ of $\mathrm{Na}^{+}$by retaining it on the side of the draw solution during the osmotic-pressure-driven permeation process. This high $\mathrm{Na}^{+}$ rejection ability makes the membrane beneficial for applications such as water desalination, and for the removal of toxic ions and organic pollutants from water through forward osmosis processes.

\section{Clean-water intake from wastewaters}

Inspired by its good $\mathrm{Na}^{+}$rejection and water permeation, the $\mathrm{F}$ CGO3 membrane was used to intake clean-water from solutions 
contaminated with organic dyes, such as methylene orange (MO) and rhodamine B (RB). As Fig. 5a shows, a noticeable liquid level difference appeared in the U-shaped tube between the $\mathrm{NaCl}$ solution $\left(2.0 \mathrm{~mol} \mathrm{~L}^{-1}\right)$ and the wastewaters, which indicates the intake of water by the $\mathrm{NaCl}$ solution through the $\mathrm{F}$ CGO3 membrane. The MO and RB pollutants were rejected by the membrane, as evidenced by the colourless $\mathrm{NaCl}$ solution after testing. In addition, after the membrane was immersed for 15 days in the high saline water and polluted solutions, the FCGO3 membranes were able to withstand the high liquid level difference between the draw and feed solutions, showcasing their excellent durability and hydraulic stability. The water intake efficiency (water permeation ratio) of the F-CGO3 membrane was determined using the same setup as the semipermeability tests shown in Fig. $\mathrm{S} 1, \dagger$ where a $2.0 \mathrm{~mol} \mathrm{~L}^{-1}$ $\mathrm{NaCl}$ solution and wastewater were used as the draw and feed solutions, respectively.

Fig. 5b shows that the initial membrane water permeation rates in MO and $\mathrm{RB}$ solutions were 12.2 and $19.3 \mathrm{~L} \mathrm{~m}^{-2} \mathrm{~h}^{-1}$, respectively. These rates decreased as the permeation time was prolonged, and reached stability after a $3 \mathrm{~h}$ permeation period.
This is due to the interaction forces and steric effects of the pollutants with the F-CGO3 membrane. As it is known, MO and $\mathrm{RB}$ have conjugated aromatic structures (Fig. S11 $\dagger$ ), and are attracted to GO sheets via the $\pi-\pi$ interaction. Additionally, coulombic interactions may also contribute to the water permeation decline because of the negatively charged GO sheets and positively charged RB. Although RB has a larger conjugated aromatic structure and more favourable coulombic interactions with the GO sheets, its water intake efficiency is higher than that of the MO solution at the same feeding weight concentration. This might be caused by the smaller molecular weight of MO, which results in a higher osmotic potential at the same weight concentration. On the other hand, the smaller molecular size of the MO molecule allows it to easily access the inlets of the interlaminar membrane channels and block the permeation of water. Furthermore, to evaluate the influence of the supporting layer on the water intake efficiency of the membranes, in Fig. 5b the water permeation rates of the F-CGO3 and S-CGO membranes in various wastewaters are compared. The figure shows that when the S-CGO3 membrane was used as the separator, the water permeation rates from feed solutions was
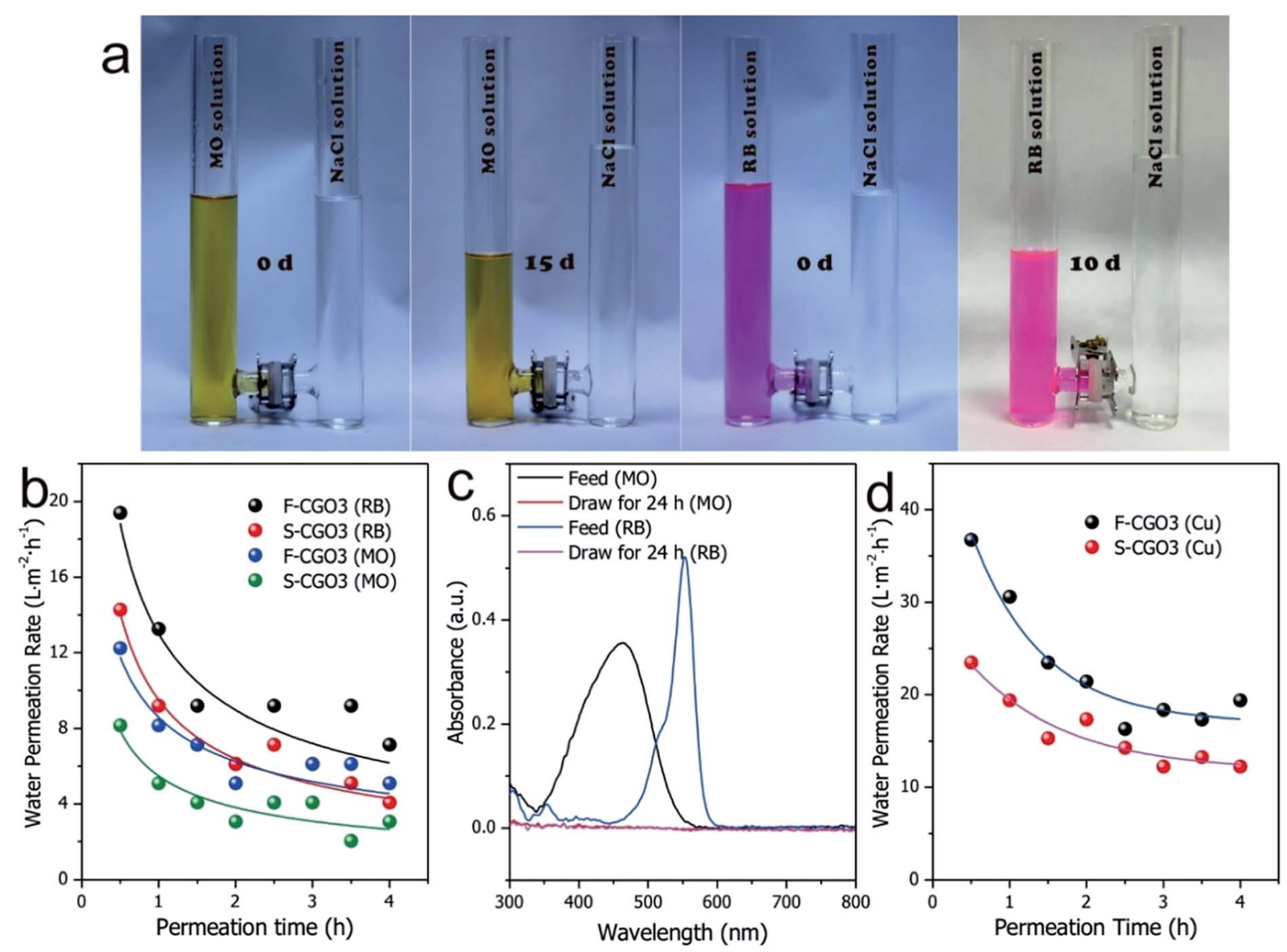

Fig. 5 (a) Digital pictures of the water permeation behaviour from a 10 ppm MO solution (left two) and a 10 ppm RB solution (right two) to a 2 mol $\mathrm{L}^{-1} \mathrm{NaCl}$ solution through the F-CGO3 membrane, which was fixed in the tunnel of a glass communicating vessel. (b) Water permeation rates of the F-CGO3 and S-CGO3 membranes in the MO or RB solution. (c) UV-Vis spectra of the original MO or RB solutions (diluted 2 times), and the corresponding draw solutions after a $24 \mathrm{~h}$ permeation period performed with the F-CGO3 membrane. (d) Water permeation rate of the $\mathrm{F}$-CG and

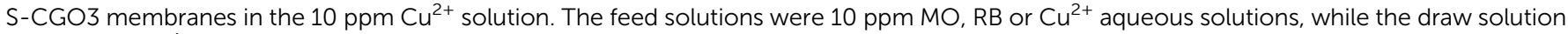
was a $2 \mathrm{~mol} \mathrm{~L}^{-1} \mathrm{NaCl}$ solution. 
lower at the beginning and during the stable interval. These results are consistent with those in Fig. 4c, thus indicating the presence of ICP effects on the permeation of water during a concentration gradient driven by a membrane separation process, such as FO. We also detected by UV-Vis spectroscopy that pollutants leaked into the draw solution. The UV-Vis spectra in Fig. 5c indicate that the absorption of MO and RB molecules are both nil, which confirms that the intake from the wastewaters is clean-water.

We also investigated the water intake efficiency of membranes from heavy metal ions solutions using $\mathrm{Cu}^{2+}$ as a model (Fig. 5d). From the results, the initial water permeation rate in a $\mathrm{Cu}^{2+}$ solution was found to reach $36.7 \mathrm{~L} \mathrm{~m}^{-2} \mathrm{~h}^{-1}$, and after $3 \mathrm{~h}$, this value stabilized to about $20 \mathrm{~L} \mathrm{~m}^{-2} \mathrm{~h}^{-1}$. This decrease in the water permeation rate could be owing to the strong coordination interactions between $\mathrm{Cu}^{2+}$ and the oxidized region of the GO sheets, which causes the $\mathrm{Cu}^{2+}$ to concentrate around the inlets of the inter-laminar channels of the F-CGO3 membrane, and results in a high water permeation resistance across the membrane, similar to what was reported by Shi et al. ${ }^{46}$ However, both the initial and stable water permeation rates obtained for the $\mathrm{Cu}^{2+}$ solution are larger than those obtained for the MO and RB solutions. This reflects the higher water intake efficiency from inorganic wastewater by the F-CGO membrane. The draw solution after permeation testing was also examined using an ICP-AES instrument. In fact, there was no $\mathrm{Cu}^{2+}$ was detected in the draw solution after permeation testing, which indicates that the heavy metal ions, i.e. $\mathrm{Cu}^{2+}$, were completely blocked by the F-CGO3 membrane. Therefore, we can conclude that the F-CGO3 membrane can be used to intake clean-water from wastewater by effectively rejecting heavy metal ions and organic pollutants. This performance is useful for the on-site water purification from natural or polluted waters, and therefore the fabricated membrane would be a promising candidate to serve as the functional membrane of some portable water purification devices, such as a military hydration bag for its use during rough missions.

\section{Conclusions}

Through the thermally-induced chemical cross-linking process, we successfully prepared a thin freestanding GO-based FO membranes with controllable inter-laminar distance and good hydraulic stability. Comparisons between the permeation performances of the membranes confirmed that the water permeation rate of the freestanding membrane is far higher than that of standing membrane, which suggests that the ICP effect induced by the FO process can be alleviated, and even eliminated, by removing the porous supporting layer. The water intake tests from water contaminated with heavy metal ion and organic dyes demonstrated that the thin freestanding GO-based FO membrane can be used to intake clean-water from wastewater by effectively rejecting heavy metal ions and organic pollutants. These properties make the fabricated membrane a promising material for the assembly of portable water purification systems that can be used by field workers and the military, or to supply drinking water during emergency situations.

\section{Acknowledgements}

This work was financially supported by the National Natural Science Foundation of China (Grants No. 11305248 and 11605274), and Shanghai Municipal Commission for Science and Technology (Grant No. 15ZR1448800).

\section{Notes and references}

1 R. R. Nair, H. A. Wu, P. N. Jayaram, I. V. Grigorieva and A. K. Geim, Science, 2012, 335, 442-444.

2 R. K. Joshi, P. Carbone, F. C. Wang, V. G. Kravets, Y. Su, I. V. Grigorieva, H. A. Wu, A. K. Geim and R. R. Nair, Science, 2014, 343, 752-754.

3 P. Sun, H. Liu, K. Wang, M. Zhong, D. Wu and H. Zhu, Chem. Commun., 2015, 51, 3251-3254.

4 P. Sun, F. Zheng, M. Zhu, Z. Song, K. Wang, M. Zhong, D. Wu, R. B. Little, Z. Xu and H. Zhu, ACS Nano, 2014, 8, 850-859.

5 P. Sun, M. Zhu, K. Wang, M. Zhong, J. Wei, D. Wu, Z. Xu and H. Zhu, ACS Nano, 2013, 7, 428-437.

6 J.-Q. Huang, T.-Z. Zhuang, Q. Zhang, H.-J. Peng, C.-M. Chen and F. Wei, ACS Nano, 2015, 9, 3002-3011.

7 K. W. Putz, O. C. Compton, M. J. Palmeri, S. T. Nguyen and L. C. Brinson, Adv. Funct. Mater., 2010, 20, 3322-3329.

8 L. Huang, Y. Li, Q. Zhou, W. Yuan and G. Shi, Adv. Mater., 2015, 27, 3797-3802.

9 T. M. Yeh, Z. Wang, D. Mahajan, B. S. Hsiao and B. Chu, J. Mater. Chem. A, 2013, 1, 12998-13003.

10 H. M. Hegab and L. Zou, J. Membr. Sci., 2015, 484, 95-106.

11 B. Liang, W. Zhan, G. Qi, S. Lin, Q. Nan, Y. Liu, B. Cao and K. Pan, J. Mater. Chem. A, 2015, 3, 5140-5147.

12 P. Lu, S. Liang, T. Zhou, X. Mei, Y. Zhang, C. Zhang, A. Umar and Q. Wang, RSC Adv., 2016, 6, 56599-56609.

13 Y. Han, Z. Xu and C. Gao, Adv. Funct. Mater., 2013, 23, 36933700.

14 H. Huang, Z. Song, N. Wei, L. Shi, Y. Mao, Y. Ying, L. Sun, Z. Xu and X. Peng, Nat. Commun., 2013, 4, 2979.

15 J.-G. Gai, X.-L. Gong, W.-W. Wang, X. Zhang and W.-L. Kang, J. Mater. Chem. A, 2014, 2, 4023-4028.

16 C.-N. Yeh, K. Raidongia, J. Shao, Q.-H. Yang and J. Huang, Nat. Chem., 2015, 7, 166-170.

17 R. K. Joshi, S. Alwarappan, M. Yoshimura, V. Sahajwalla and Y. Nishina, Appl. Mater. Today, 2015, 1, 1-12.

18 L. J. Cote, J. Kim, Z. Zhang, C. Sun and J. Huang, Soft Matter, 2010, 6, 6096-6101.

19 S. Sun, C. Wang, M. Chen and M. Li, Chem. Phys. Lett., 2013, 561, 166-169.

20 Z. Jia and W. Shi, Carbon, 2016, 101, 290-295.

21 Z. Jia and Y. Wang, J. Mater. Chem. A, 2015, 3, 4405-4412.

22 S. Park, K.-S. Lee, G. Bozoklu, W. Cai, S. T. Nguyen and R. S. Ruoff, ACS Nano, 2008, 2, 572-578.

23 H. Liu, H. Wang and X. Zhang, Adv. Mater., 2015, 27, 249254.

24 P. Sun, Q. Chen, X. Li, H. Liu, K. Wang, M. Zhong, J. Wei, D. Wu, R. Ma, T. Sasaki and H. Zhu, NPG Asia Mater., 2015, 7, e162. 
25 P. Sun, R. Ma, W. Ma, J. Wu, K. Wang, T. Sasaki and H. Zhu, NPG Asia Mater., 2016, 8, e259.

26 P. Nasr and H. Sewilam, Clean Technol. Environ., 2015, 17, 2079-2090.

27 M. Xie, J. Lee, L. D. Nghiem and M. Elimelech, J. Membr. Sci., 2015, 493, 748-754.

28 S. Zhao, L. Zou, C. Y. Tang and D. Mulcahy, J. Membr. Sci., 2012, 396, 1-21.

29 B. Mi and M. Elimelech, J. Membr. Sci., 2010, 348, 337-345.

30 Y. Huang, H. Jin, H. Li, P. Yu and Y. Luo, RSC Adv., 2015, 5, 106113-106121.

31 M. Daly, C. Cao, H. Sun, Y. Sun, T. Filleter and C. V. Singh, ACS Nano, 2016, 10, 1939-1947.

32 Y. Gao, L.-Q. Liu, S.-Z. Zu, K. Peng, D. Zhou, B.-H. Han and Z. Zhang, ACS Nano, 2011, 5, 2134-2141.

33 C. Yu, B. Zhang, F. Yan, J. Zhao, J. Li, L. Li and J. Li, Carbon, 2016, 105, 291-296.

34 J. Zhao, B. Zhang, C. Yu, Y. Liu, W. Wang and J. Li, Carbon, 2016, 109, 487-494.

35 S. Xie, J. Zhao, B. Zhang, Z. Wang, H. Ma, C. Yu, M. Yu, L. Li and J. Li, ACS Appl. Mater. Interfaces, 2015, 7, 17558-17564.
36 B. Zhang, Y. Zhang, C. Peng, M. Yu, L. Li, B. Deng, P. Hu, C. Fan, J. Li and Q. Huang, Nanoscale, 2012, 4, 1742-1748.

37 G. Jiang, M. Goledzinowski, F. J. E. Comeau, H. Zarrin, G. Lui, J. Lenos, A. Veileux, G. Liu, J. Zhang, S. Hemmati, J. Qiao and Z. Chen, Adv. Funct. Mater., 2016, 26, 1729-1736. 38 H. Wang, J. T. Robinson, X. Li and H. Dai, J. Am. Chem. Soc., 2009, 131, 9910-9911.

39 B. Zhang, L. Li, Z. Wang, S. Xie, Y. Zhang, Y. Shen, M. Yu, B. Deng, Q. Huang, C. Fan and J. Li, J. Mater. Chem., 2012, 22, 7775-7781.

40 P. Y. Han, M. Tani, M. Usami, S. Kono, R. Kersting and X. C. Zhang, J. Appl. Phys., 2001, 89, 2357-2359.

41 C. Ronne and S. R. Keiding, J. Mol. Liq., 2002, 101, 199-218. 42 J. T. Kindt and C. A. Schmuttenmaer, J. Phys. Chem., 1996, 100, 10373-10379.

43 B. Hussain, M. Ahmed, M. Nawaz, M. Saleem, M. Razzaq, M. A. Zia and M. Iqbal, Appl. Opt., 2012, 51, 5326-5330.

44 H. N. Ritland, J. Am. Ceram. Soc., 1955, 38, 86-88.

45 D. Mergel, D. Buschendorf, S. Eggert, R. Grammes and B. Samset, Thin Solid Films, 2000, 371, 218-224.

46 J. Liu, G. S. Shi, P. Guo, J. R. Yang and H. P. Fang, Phys. Rev. Lett., 2015, 115, 164502. 